\title{
The values of applying classification and counts of white blood cells to the prognostic evaluation of resectable gastric cancers
}

Yin-Ling Wang ${ }^{1+}$, Xin-Xin Ge ${ }^{1 \dagger}$, Yi Wang ${ }^{1 \dagger}$, Meng-Dan Xu' , Fei-Ran Gong ${ }^{2}$, Min Tao ${ }^{1,3}$, Wen-Jie Wang ${ }^{1,4}$, Liu-Mei Shou ${ }^{5}$, Kai Chen ${ }^{1 *}$, Meng-Yao Wu ${ }^{1 *}$ and Wei $\mathrm{Li}^{1,3,6^{*}}$ (D)

\begin{abstract}
Background: The classifications and counts of white blood cells (WBCs) have been proved to be able to be used as prognostic markers in cancer cases. The present study investigated the potential values of the classifications and counts of WBC, including lymphocyte (LY), monocyte (MO), neutrophil (NE), eosinophil (EO), and basophil (BA) in the prognosis of resectable gastric cancers (GCs).

Methods: This retrospective study recruited 104 resectable GC cases which were pathologically confirmed. The patients were divided into two groups according to the median pre-treatment values. To evaluate the changes in WBC counts and classification after treatment, we introduced the concept of post/pre-treatment ratios $(\leq 1$ indicated count was not increased after therapy, while $>1$ suggested increased count).

Results: Pre-treatment NE and total WBC counts were negatively correlated with overall survival (OS). Surgery significantly decreased the level of NE count, but increased the level of EO, whereas had no effect on the levels of LY, MO, BAor total WBC. Adjuvant chemotherapy significantly decreased the level of BA. Whole course of treatment (surgery combined with adjuvant chemotherapy) had no significant effect on the counts of $L Y, M O, N E$, EO, BA or total WBC. Post/pre-treatment ratios of LY, MO NE, EO, BA and total WBC levels had no effects on OS. Univariate analysis indicated that AJCC stage (III) and higher level of pre-treatment total WBC count were prognostic factors affecting OS. Multivariate Cox regression analysis revealed that AJCC stage (III) and higher level of pre-treatment total WBC count were independent prognostic factors.
\end{abstract}

Conclusions: Pre-treatment NE count and pre-treatment total WBC count may be potential prognostic factors for the prognostic evaluation of GCs.

Keywords: Prognosis, Overall survival (OS), White blood cell (WBC), Gastric cancer (GC)

\section{Background}

Gastric cancer (GC) is the fourth most common cancer in the world and the second essential factor to death of cancers $[1,2]$. According to the cancer statistics in 2017, new gastric cancer cases numbered almost 28,000 , and more than 10,960 deaths were caused by gastric cancer

\footnotetext{
* Correspondence: kaichen@suda.edu.cn; mywu@suda.edu.cn;

liwei10@suda.edu.cn

†Yin-Ling Wang, Xin-Xin Ge and Yi Wang contributed equally to this work. ${ }^{1}$ Department of Oncology, the First Affiliated Hospital of Soochow University, Suzhou 215006, China

Full list of author information is available at the end of the article
}

in united states [2]. Although both the lethality and morbidity of GC have decreased in recent years, the 5-year survival rate remains quite low still [3]. Therefore, it is important to identify reliable predictive factors for the prognosis of GC patients.

Total white blood cell (WBC) count which is often elevated during infections, is one of the nonspecific markers of inflammation and can be linked with some particular kinds of cancers [4]. WBC can be divided into five types, including lymphocyte (LY), monocyte (MO), neutrophil (NE), eosinophil (EO), basophil (BA) [5]. LY

(C) The Author(s). 2018 Open Access This article is distributed under the terms of the Creative Commons Attribution 4.0 International License (http://creativecommons.org/licenses/by/4.0/), which permits unrestricted use, distribution, and 
can respond to the host's immune response, and low level of LY has been reported in various cancers $[6,7]$. $\mathrm{MO}$ is produced in the bone marrow and stored in bone marrow, liver, as well as lymph nodes. MO plays critical role in systemic inflammatory response and steady-state immune-surveillance [8]. NE responds to infection and injury [9]. In addition, an elevated count of blood NE has been shown to predict poor survival in advanced GC [10] and esophageal cancer [11]. Besides, Sam C. Wang et al. proved that pretreatment NE to LY ratio independently predicts disease-specific survival in patients with resectable gastroesophageal junction and gastric adenocarcinoma [12]. EO is uncommon in healthy individuals, however, it is associated with allergies, helminth infections and some inflammatory states. Moreover, previous studies have shown a close association between EO level and the prognosis of GC patients [13]. BA, derived from bone marrow, is the rarest granulocytes and occupied less than $1 \%$ of peripheral leukocytes [14]. BA is well recognized to play a crucial role in protection against infections with parasites, allergy and autoimmunity $[15,16]$.

The present study investigated whether the classification and counts of WBCs can be served as prognostic indicators in patients with the resectable GC.

\section{Methods}

\section{Subjects and inclusion criteria}

This study was conducted as a retrospective investigation of resectable GC patients that had been referred to the First Affiliated Hospital of Soochow University (Jiangsu, China) between June 2007 and July 2016. Approval for the study was granted by the Medical Ethics Committees of the First Affiliated Hospital of Soochow University. Clinical and pathological records of all the patients participating in the study were reviewed periodically.

In total, 104 resectable GC patients were recruited in this study. All cases were confirmed by surgery and pathology. Patient characteristics are detailed in Table 1. The median age of the 104 patients was 60 years (range, 30-77 years). 77 patients were male and 27 were female. The staging of cancer was made according to tumor-nodulus-metastases (TNM) classification and classified through the American Joint Committee on Cancer (AJCC) recommendations. The prognostic analyses were performed regarding overall survival (OS).

\section{Blood samples}

Peripheral venous blood $(5-7 \mathrm{ml})$ was collected into a sterile ethylenediaminetetraacetic acid (EDTA) tube. All blood samples were fasted and obtained between 6:30 and 7:30 a.m. in order to standardize the known impact of circulating hormones (circadian rhythm) on the number and subtype distribution of the various WBC indices.
Hematological parameters were analyzed within 30 min after collection using a hematology analyser (Sysmex XE-2100; Sysmex, Kobe, Japan). LY, MO, EO, NE, BA and total WBC counts were recorded in Table 1 . The patients were divided into two groups according to the median counts of LY, MO, EO, NE, BA or total WBC. The post/pre-treatment ratios were defined as the rate of pre-therapy blood parameters count and the corresponding ones obtained after therapy.

\section{Evaluation}

Computed tomography (CT) scan was performed for the assessment of response every 2 months and evaluated according to the criteria of Response Evaluation Criteria in Solid Tumors (RECIST) 1.1.

\section{Follow-up}

Survival time was measured from the date of chemotherapy until death or last clinical evaluation. The prognostic analyses were performed regarding OS. OS was defined as the time from the diagnosed date to death from any cause.

\section{Statistical analysis}

All statistical analyses were performed using SPSS 19.0 software (Chicago, USA). For analysis of survival data, Kaplan-Meier curves were constructed, and statistical analysis was carried out using the log-rank test. The associations between blood parameters status and clinicopathologic features were explored by the $\mathrm{x} 2$ tests. The relationships between changes in the blood parameters status and treatments were assessed by the $t$ tests. Multivariate logistic regression model was employed to identify the independent risk factors associated with resectable GC. All values of $P<0.05$ were considered statistically significant.

\section{Results}

The clinicopathologic features of resectable GC patients

Table 1 summarizes the clinicopathological characteristics and their relationship to the levels of LY, MO, NE, $\mathrm{EO}, \mathrm{BA}$ and total $\mathrm{WBC}$. The gender was significantly different in levels of $\operatorname{NE}(P=0.004), \operatorname{EO}(P=0.044)$, $\mathrm{BA}(P=0.005)$ and total $\mathrm{WBC}(P=0.044)$ compared to those of LY and MO (both $P>0.05$ ). The age was significantly different in levels of BA $(P=0.034)$ compared to those of LY, MO, NE, EO and total WBC (all $P>0.05$ ). The tumor size was significantly different in levels of LY $(P=0.039)$ compared to those of MO, NE, EO,BA and total WBC (all $P>0.05$ ). The depth of invasion was significantly different in levels of $\mathrm{NE}$ $(P=0.028)$ compared to those of LY, MO, EO,BA and total WBC (all $P>0.05$ ). The Lymphonodus metastasis was significantly different in levels of NE and total WBC (both $P=0.027$ ) compared to those of LY, MO, 


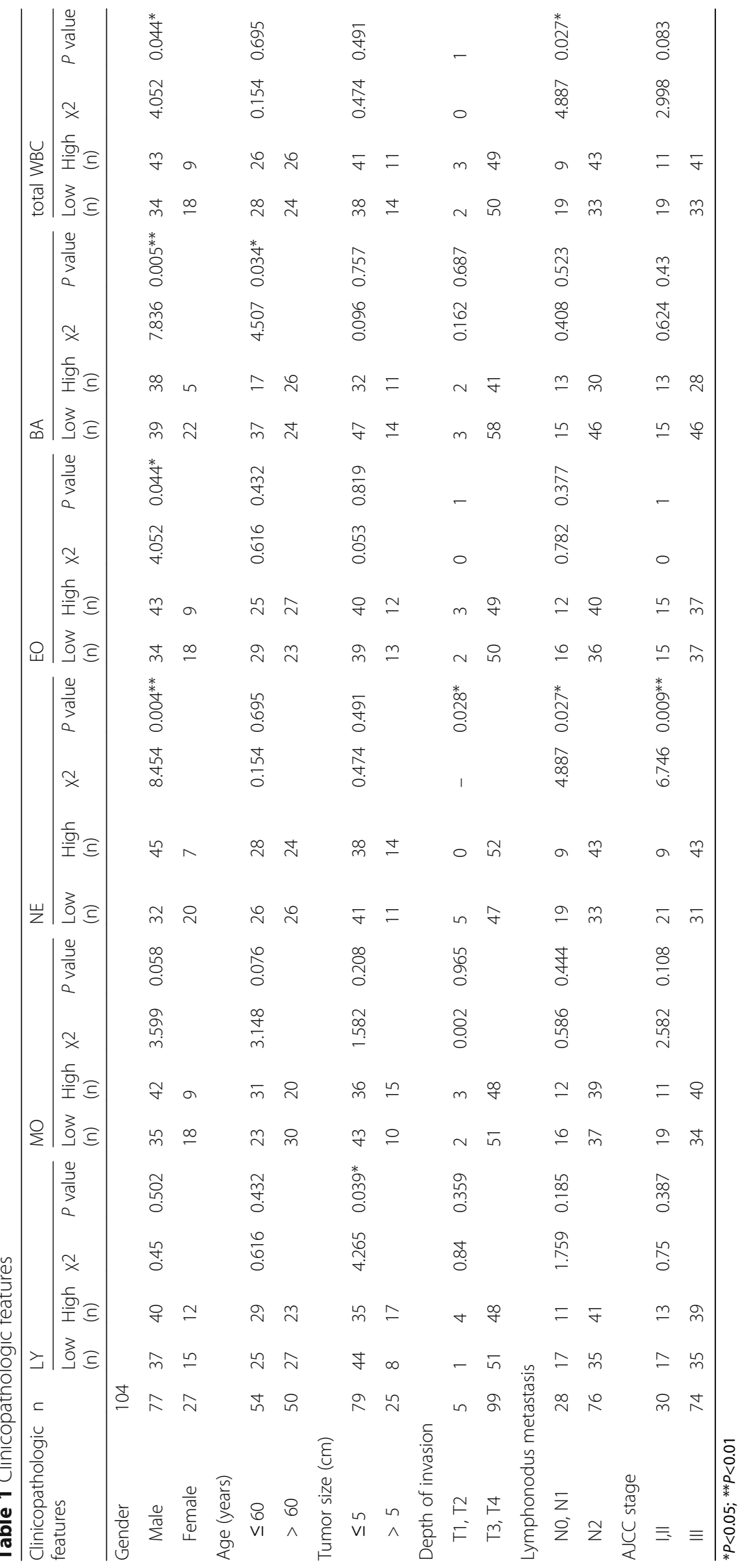


EO and BA (all $P>0.05$ ). The AJCC stage was significantly different in levels of NE $(P=0.009)$ compared to those of LY, MO, EO,BA and total WBC (all $P>0.05$ ).

\section{Pre-treatment NE and total WBC count were correlated with outcomes of resectable GC patients}

The Kaplan-Meier plots were used to determine the relationship between $\mathrm{OS}$ and the status of pre-treatment LY, MO, NE, EO, BA and total WBC (Fig. 1a-f). The patients were divided into two groups according to the median values of pre-treatment LY (low LY, $\leq 1.535 \times 10^{9} / \mathrm{L}$ or high $\mathrm{LY},>1.535 \times 10^{9} / \mathrm{L}$ ), pre-treatment $\mathrm{MO}$ (low $\mathrm{MO}, \leq 0.330 \times 10^{9} / \mathrm{L}$ or high $\left.\mathrm{MO},>0.330 \times 10^{9} / \mathrm{L}\right)$, pre-treatment NE (low NE, $\leq 3.325 \times 10^{9} / \mathrm{L}$ or high $\mathrm{NE}$, $>3.325 \times 10^{9} / \mathrm{L}$ ), pre-treatment EO (low EO, $\leq 0.090 \times$ $10^{9} / \mathrm{L}$ or high $\left.\mathrm{EO},>0.090 \times 10^{9} / \mathrm{L}\right)$, pre-treatment $\mathrm{BA}$ (low $\mathrm{BA}, \leq 0.010 \times 10^{9} / \mathrm{L}$ or high $\mathrm{BA},>0.010 \times 10^{9} / \mathrm{L}$ ) or pre-treatment total WBC (low WBC, $\leq 5.361 \times 10^{9} / \mathrm{L}$ or high $\left.\mathrm{WBC},>5.361 \times 10^{9} / \mathrm{L}\right)$. The median OS of the high LY group was 26 (95\% confidence interval [CI] 23.90528.095) months, while that of the low LY group was 30 (95\% CI 27.352-32.648) months $(P=0.050)$. The median OS of the high NE group was 26 (95\% CI 23.35228.648) months, while that of the low NE group was 29 (95\% CI 23.952-34.048) months $(P=0.020)$. The median OS was 26 (95\% CI 24.400-27.600) months in the high WBC group and 31 (95\% CI 25.952-36.048) months in the low WBC group $(P=0.001)$. The median OS in $\mathrm{MO}$, EO and BA has no significance(all $P>0.05)$ Thus, higher pre-treatment NE and total WBC levels were correlated with poorer prognosis. And pre-treatment levels of LY, $\mathrm{MO}, \mathrm{EO}$ or BA had no significant effects on OS.

\section{Effects of surgery on the counts of LY, MO, NE, EO, BA and total WBC}

The effects of surgery on the counts of, LY, MO, NE, EO, BA and total WBC were presented in Fig. 2a-f, respectively. The median count of NE was $3.325 \times 10^{9} / \mathrm{L}(95 \% \mathrm{CI}$ $\left.2.870 \times 10^{9} / \mathrm{L}-3.535 \times 10^{9} / \mathrm{L}\right)$ before surgery, and $2.910 \times$ $10^{9} / \mathrm{L}\left(95 \%\right.$ CI $\left.2.540 \times 10^{9} / \mathrm{L}-3.145 \times 10^{9} / \mathrm{L}\right)$ after surgery $(P=0.043)$. The median count of EO was $0.090 \times 10^{9} / \mathrm{L}$ $\left(95 \%\right.$ CI $\left.0.080 \times 10^{9} / \mathrm{L}-0.100 \times 10^{9} / \mathrm{L}\right)$ before surgery, and $0.110 \times 10^{9} / \mathrm{L}\left(95 \%\right.$ CI $\left.0.100 \times 10^{9} / \mathrm{L}-0.140 \times 10^{9} / \mathrm{L}\right)$ after surgery $(P=0.022)$. The median count of total WBC and several other WBC like LY, MO and BA has no statistical difference between before surgery and after surgery (all $P>0.323$ ). Surgery decreased the count of NE, but increased the count of EO, whereas had no significant impact on the count of LY, MO, BA or total WBC.

Effects of adjuvant chemotherapyon the counts of LY, MO, NE, EO, BA and total WBC

The effects of adjuvant chemotherapy on the counts of LY, MO, NE, EO BA and total WBC were presented in
Fig. 3a-f. Only the median count of BA was significant between the before adjuvant chemotherapy group $(0.010 \times$ $10^{9} / \mathrm{L}, 95 \%$ CI $\left.0.010 \times 10^{9} / \mathrm{L}-0.010 \times 10^{9} / \mathrm{L}\right)$ and the after adjuvant chemotherapy group $\left(0.010 \times 10^{9} / \mathrm{L}, 95 \%\right.$ CI $\left.0.010 \times 10^{9} / \mathrm{L}-0.010 \times 10^{9} / \mathrm{L}\right) \quad(P=0.049)$. The median count of total WBC and other WBC such as LY, MO, NE and EO have no significance(all $P>0.05)$. Adjuvant chemotherapy decreased the count of BA, whereas had no significant impact on the count of LY, MO, NE, EO or total WBC.

\section{Effects of whole course of treatment on the counts of $L Y$, MO, NE, EO, BA and total WBC}

The impacts of whole course of treatment (surgery and adjuvant chemotherapy) on the count of LY, MO, NE, EO BA and total WBC were presented in Fig. 4a-f. No matter in before treatment group or after treatment group, there is no significant change in the count of LY, MO, NE, BA and total WBC(all $P>0.05)$, which means whole course of treatment (surgery combined with adjuvant chemotherapy) had no significant effect.

\section{Changes in LY, MO NE, EO, BA and total WBC levels after whole course of treatment could not predict outcomes of resectable GC patients}

The Kaplan-Meier plots were used to determine the effect of changes in the blood parameters status for OS (Fig. 5a-f). The median OS of patients whose LY levels increased following whole course of treatment were 28 (95\% CI 25.778-30.222) months, while that of the not-increased LY group was 26 (95\% CI 21.818-30.182) months $(P=0.374)$. The median OS of patients whose $\mathrm{MO}$ and NE levels increased following whole course of treatment were both 26 months, while that of the not-increased $\mathrm{MO}$ group and NE group were both 28 months (both $P>0.05$ ). The median OS of patients whose EO levels increased following whole course of treatment were 26 (95\% CI 23.841-28.159) months, while that of the not-increased EO group was 29 (95\% CI 25.040-32.960) months $(P=0.396)$. The median OS of patients whose BA and total WBC levels increased following whole course of treatment were both 28 months, while that of the not-increased BA group and total WBC groupwere both 28 months (both $P>0.05$ ). The median OS of patients whose WBC levels increased following whole course of treatment were 28 (95\% CI 24.617-31.383) months, while that of the not-increased WBC group was 28 (95\% CI 24.343-31.657) months $(P=0.188)$. Thus, changes in LY, MO NE, EO, BA and total WBC levels after therapy had no effects on OS.

\section{Prognostic factors for resectable GCs}

Univariate analyses demonstrated that lymphonodus metastasis (N2) (hazard ratio [HR] 2.116; 95\% CI 1.316- 

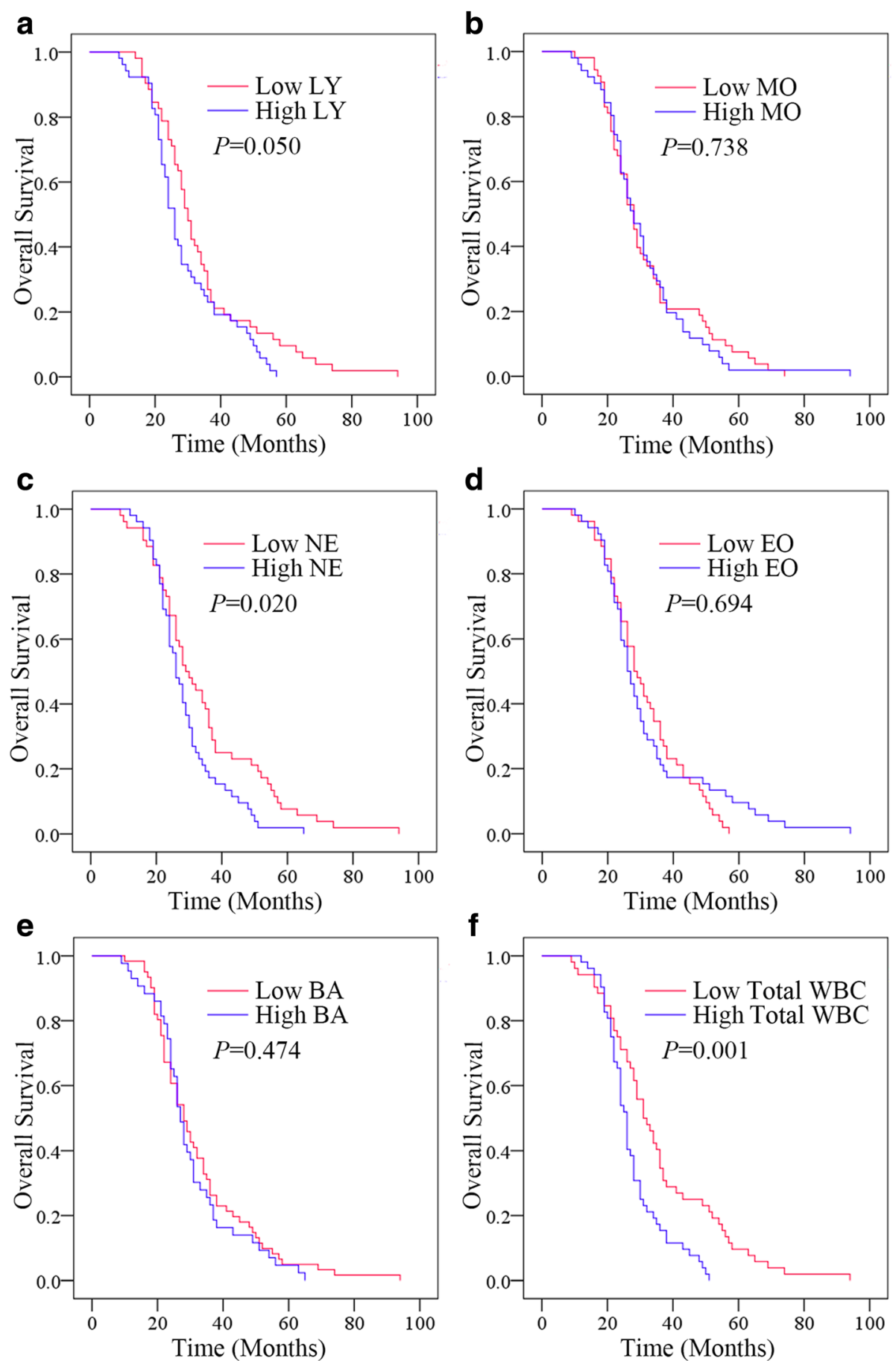

Fig. 1 Relationship between status of pre-treatment WBCs and the outcomes. a The OS according to pre-treatment LY $\mathbf{b}$ The OS according to pre-treatment MO. c The OS according to pre-treatment NE. $\mathbf{d}$ The OS according to pre-treatment EO. e The OS according to BA. $\mathbf{f}$ The OS according to pre-treatment total WBC

3.403; $P=0.002)$, AJCC stage (III) (HR 2.764; $95 \%$ CI 1.641-4.657; $P=0.000)$, higher pre-treatment NE level (HR 1.585; 95\% CI 1.060-2.371; $P=0.025)$ and higher pre-treatment WBC level (HR 2.023; 95\% CI 1.3293.081; $P=0.001)$ were significant risk factors for a poor prognosis (Table 2). In multivariate analysis, AJCC stag (III) (HR 4.199; 95\% CI 1.237-14.247; $P=0.021$ ) and higher pre-treatment total WBC count (HR 1.751; 95\% CI $1.045-2.935 ; P=0.033$ ) were found to be independently associated with poor survival. 

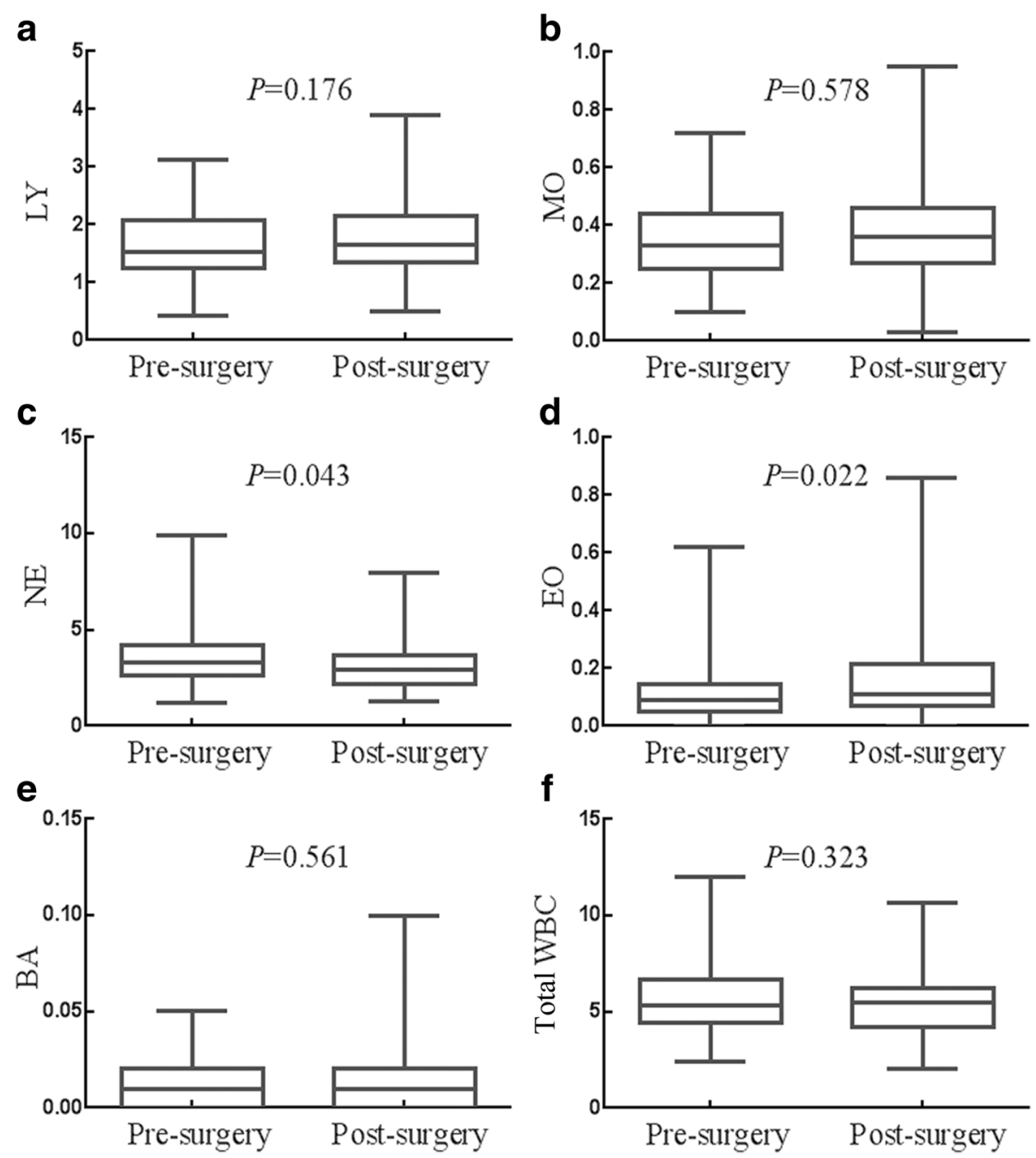

Fig. 2 Effects of surgery on the status of WBCs. a Surgery had no influence on the count of LY. $\mathbf{b}$ Surgery had no influence on the count of MO. c Surgery decreased the count of NE. $\mathbf{d}$ Surgery increased the count of EO. e Surgery had no influence on the count of BA. f Surgery had no influence on the count of total WBC

\section{Discussion}

To date, many studies have reported the relationship between the elevated total WBC count and cancer risks. Thomas P. Erlinger et al. proved that WBC is associated with total cancer mortality after adjustment for age, sex and race [17]. Yong-Jae Lee and colleagues identified that increased WBC count was associated with a higher mortality and incidence risk of colon cancer [18]. Masahiro Lida et al. found that the incidence of GC increased linearly with increasing count of total WBC [19]. Moreover, total WBC count was negatively related to the survival of GC, especially with $H$. pylori (HP) infection [19]. $H$. pylori infection has been shown to increase the production of reactive oxygen metabolites [20,21], which often cause extensive tissue damage and DNA damage, leading to mutations of oncogenes and tumor suppressors $[22,23]$. Therefore, the relationship between total
WBC count and survival of GC could be a reflection of the extent of mucosal inflammation induced by $H$. pylori infection [24]. In our present study, we found that higher pre-treatment total WBC status was associated with worse OS, in consisting with previous studies. Univariate analysis demonstrated that higher pre-treatment total WBC count was a significant risk factors and multivariate analysis demonstrated that total $\mathrm{WBC}$ count was an independent prognostic marker for a poor prognosis. However, neither surgery nor adjuvant chemotherapy could affect total WBC count. Post/pre-treatment ratio of total WBC had no significant effects on OS either.

Previous studies have confirmed that peripheral blood LY count is an independent prognostic factor for multiple cancers, such as breast [25], pancreatic [26], lung [27], cervical [28] and gallbladder cancers [29]. In cell-mediated anti-tumor immune response, LY has a 

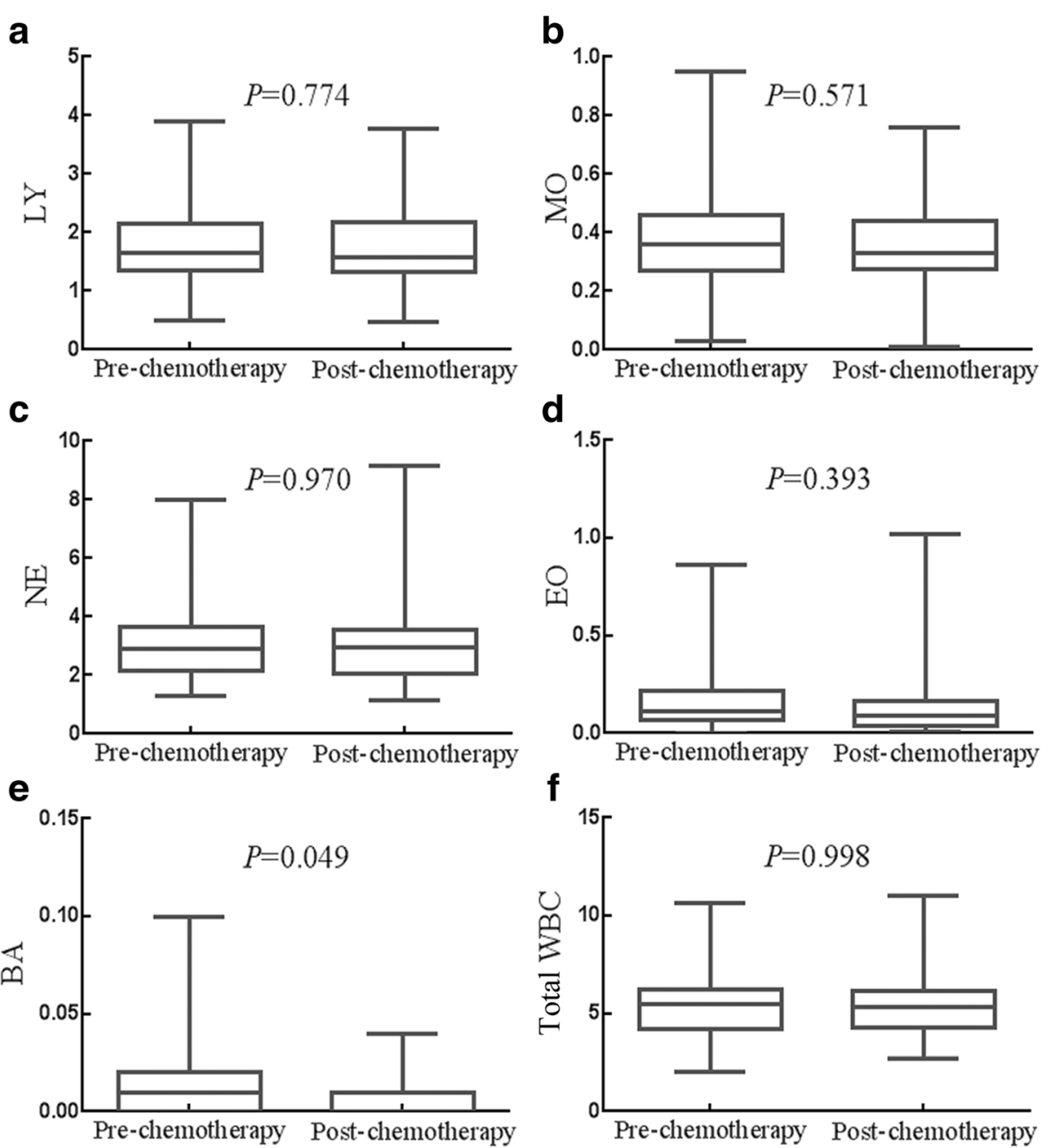

Fig. 3 Effects of adjuvant chemotherapy on the status of WBCs. a Chemotherapy had no influence on the count of LY. b Chemotherapy had no influence on the count of MO. c Chemotherapy had no influence on the count of NE. $\mathbf{d}$ Chemotherapy had no influence on the count of EO. e Chemotherapy decreased the count of BA. $\mathbf{f}$ Chemotherapy had no influence on the count of total WBC

crucial role in many cancers [30, 31]. Tumor-infiltrating lymphocytes (TILs) have a positive effect on OS in colorectal and ovarian cancers [32, 33], which may due to the apoptosis induced by tumor cells and the activation of specific CD8+ T cells. In addition, CD4+ T lymphocytes play a key role by secreting cytokines such as IL-2, which is required for CD8+ T lymphocytes proliferation and growth. Previous studies indicated that, activation of CD4+ $\mathrm{T}$ cells are required for immunization of CD8+ T cells against cancer [34]. However, in the present study, we found that LY count was not associated with OS in resectable GC patients.

Inflammation-induced carcinogenesis is caused by several processes, including genotoxicity, aberrant tissue repair, proliferation, invasion and metastasis [35]. MO, as a marker of systemic inflammatory response, may thus be a predictive factor for various inflammation-related cancers. In many previous studies, MO has been proved to be negatively related to OS of tumor patients, such as pancreatic cancer and hepatocellular carcinoma [36, 37]. Multiple mechanisms might be involved in the relationship between MO and tumor prognosis. As mentioned above, the inflammatory response has laid an important foundation in the development of cancer. MO, as an important component of the adaptive and innate immune system, can both facilitate angiogenesis and tumorigenesis. MO can also secret soluble mediators and promote the growth of cancer [38]. In addition, Evani et al. found that MO can promote proliferation of breast cancer by improving adhesion of tumor cells [39]. Besides, MO can differentiate into tumor-associated macrophage (TAM), which plays a major part in tumor microenvironment through promoting tumor progression, metastasis, angiogenesis, migration and immune escape [40]. However, in our present study, neither surgery nor adjuvant chemotherapy had significant effects on MO count. 

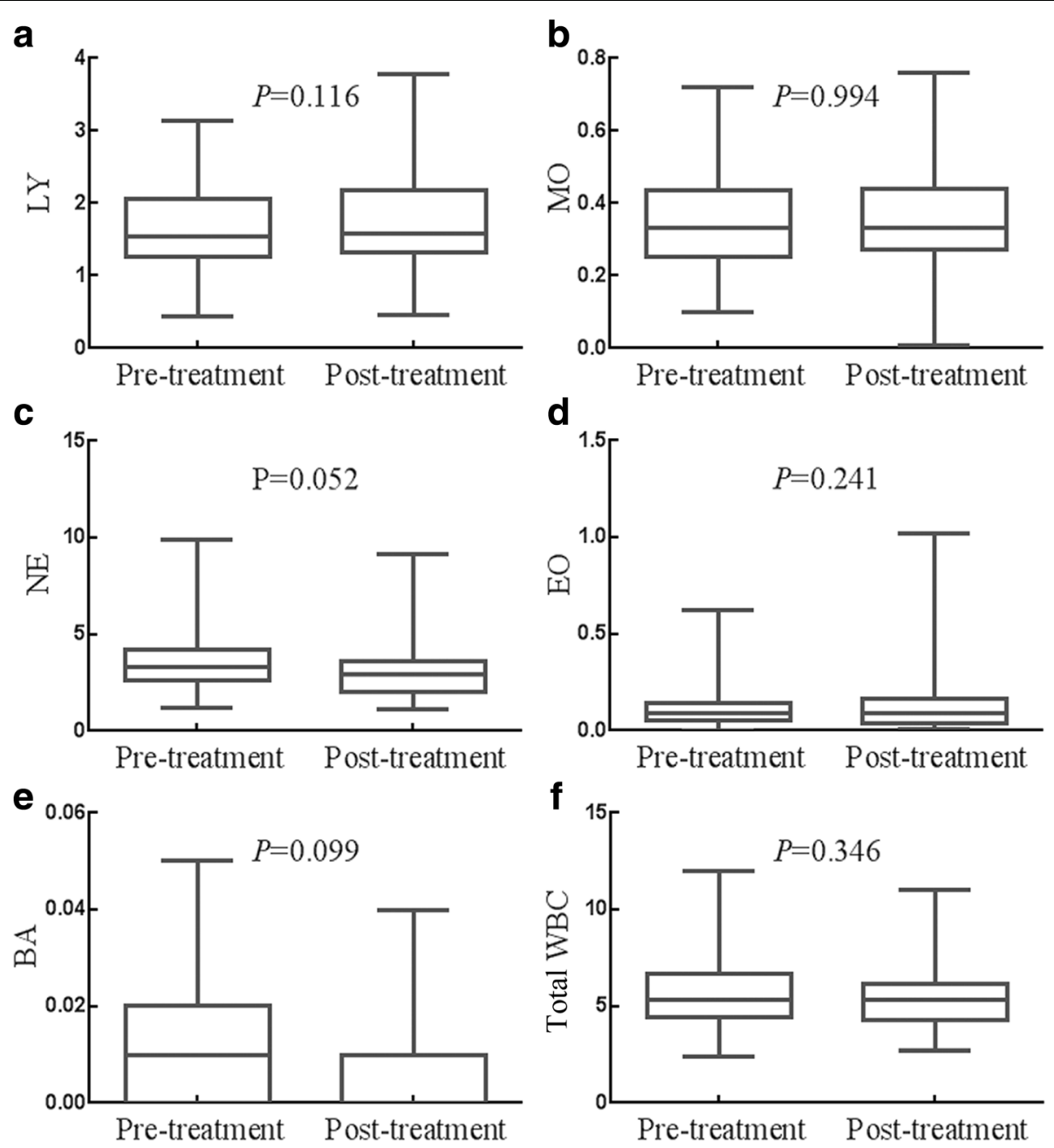

Fig. 4 Effects of whole course of treatment on the status of WBCs. a Whole course of treatment had no influence on the count of LY. $\mathbf{b}$ Whole course of treatment had no influence on the count of MO. $\mathbf{c}$ Whole course of treatment had no influence on the count of NE. (D) Whole course of treatment had no influence on the count of EO. e Whole course of treatment had no influence on the count of BA. $\mathbf{f}$ Whole course of treatment had no influence on the count of total WBC

In addition, pre-treatment or post/pre-treatment ratio of MO had no effects on OS, either.

Chronic inflammation is essential for cancer growth and metastasis. NE is one of the well-known marker of systemic inflammatory response. Increasing evidences show that it is also a predictor of poor prognosis in a variety of malignant tumors [10, 11, 41, 42]. It has been reported that activation of endogenous or exogenous pathways could mobilize transcription factors and inflammatory mediators, which may led to recruitment of inflammatory cells, including NE [43]. In our present study, we found that NE count could be down-regulated by surgery. In addition, pre-treatment NE count was negatively related to OS, and univariate analysis indicated that higher pre-treatment NE count was a significant risk factors for a poor prognosis. Basing on changes in individual NE count levels, post/pre-treatment ratio of NE had no significant effects on OS.
Higher EO count has been observed in several malignant tumors, such as colorectal [44], breast [45], cervical [46], oral squamous [47] and prostate cancers [48]. The relationship between EO and prognosis is still indefinite. In colorectal and prostate cancers, EO infiltration has been linked to a favourable prognosis $[44,48]$. However, EO appears to be an independent and significant unfavorable prognostic factor in adult $\mathrm{T}$ cell leukaemia/ lymphoma, chronic eosinophilic leukemia and Hodgkin's lymphoma [49-51]. The growth-promoting effect EO could be executed through secreting several pro-angiogenic factors, including VEGF, fibroblast growth factor-2, and IL-8 [52]. Our present study revealed that surgery increased the level of EO count. However, neither pre-treatment level nor post/pre-treatment ratio of $\mathrm{EO}$ count was related to the prognosis of resectable GC patients. 

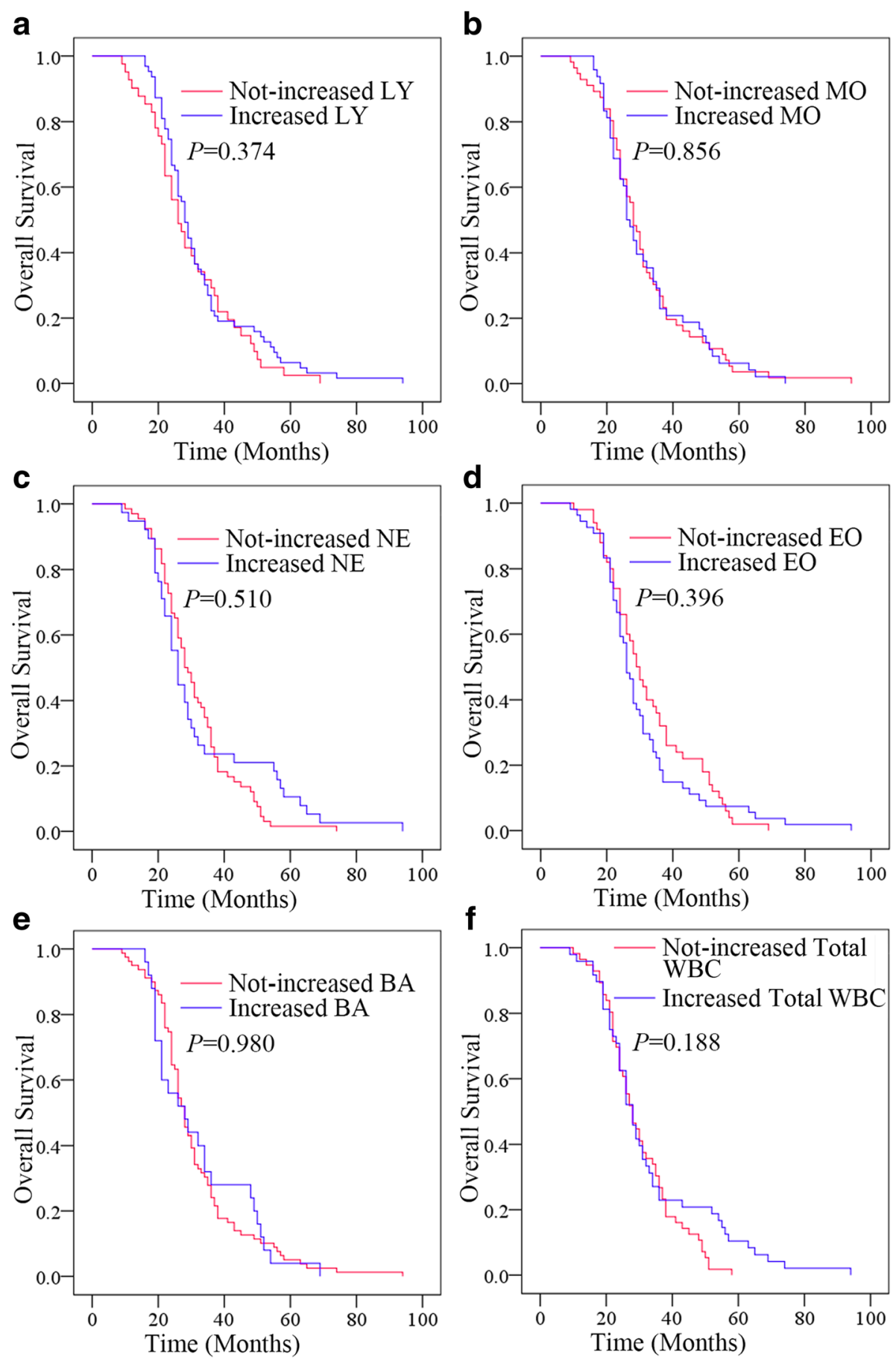

Fig. 5 Relationship between changes in status of WBCs after whole course of treatment and the outcomes. a The OS according to changes in LY. b The OS according to changes in MO. c The OS according to changes in NE. $\mathbf{d}$ The OS according to changes in EO. e The OS according to changes in BA. $\mathbf{f}$ The OS according to changes in total WBC

Researches on the functions of BA were hindered by their rarity, difficulty in identifying, and lack of useful analytical tools [14]. Existing researches have focused on the role of BA playing in parasite allergy, immediate hypersensitivity and autoimmunity [16]. Moreover, as a type of immune cells, BA is demonstrated to be negatively associated with outcomes of pancreatic cancer [53]. Additionally, in a mouse model of metastatic breast cancer by implanting $4 \mathrm{~T} 1$ cells into the mammary fat pads, some reseacheres identified that the presence of 
Table 2 Univariate and multivariate logistic regression analysis of risk factors

\begin{tabular}{|c|c|c|c|c|}
\hline \multirow[t]{3}{*}{ Risk Factors } & \multicolumn{4}{|l|}{ Overall Survival (OS) } \\
\hline & \multicolumn{2}{|l|}{ Univariate analysis } & \multicolumn{2}{|l|}{ Multivariate analysis } \\
\hline & OR $(95 \% \mathrm{Cl})$ & $P$ value & OR $(95 \% \mathrm{Cl})$ & $P$ value \\
\hline \multicolumn{5}{|l|}{ Gender } \\
\hline (Female or Male) & $0.714(0.457-1.115)$ & 0.139 & - & - \\
\hline \multicolumn{5}{|l|}{ Age } \\
\hline (> 60 years or $\leq 60$ years) & $1.015(0.992-1.038)$ & 0.192 & - & - \\
\hline \multicolumn{5}{|l|}{ Tumor size $(\mathrm{cm})$} \\
\hline$(>5$ or $\leq 5)$ & $0.848(0.538-1.337)$ & 0.478 & - & - \\
\hline Depth of invasion & $2.062(0.745-5.705)$ & 0.164 & - & - \\
\hline \multicolumn{5}{|l|}{ (T3-4 or $\mathrm{T} 1-2)$} \\
\hline \multicolumn{5}{|l|}{ Lymphonodus metastasis } \\
\hline (N2 or N0-1) & $2.116(1.316-3.403)$ & $0.002^{* *}$ & $0.544(0.166-1.777)$ & 0.313 \\
\hline \multicolumn{5}{|l|}{ AJCC stage } \\
\hline (III orl-II) & $2.764(1.641-4.657)$ & $0.000^{* *}$ & $4.199(1.237-14.24)$ & $0.021^{*}$ \\
\hline \multicolumn{5}{|l|}{ Pre-treatment LY } \\
\hline$\left(>1.535 \times 10^{9} / \mathrm{L}\right.$ or $\left.\leq 1.535 \times 10^{9} / \mathrm{L}\right)$ & $1.474(0.987-2.200)$ & 0.058 & - & - \\
\hline \multicolumn{5}{|l|}{ Pre-treatment MO } \\
\hline$\left(>0.330 \times 10^{9} / \mathrm{L}\right.$ or $\left.\leq 0.330 \times 10^{9} / \mathrm{L}\right)$ & $1.067(0.722-1.576)$ & 0.745 & - & - \\
\hline \multicolumn{5}{|l|}{ Pre-treatment NE } \\
\hline$\left(>3.325 \times 10^{9} / \mathrm{L}\right.$ or $\left.\leq 3.325 \times 10^{9} / \mathrm{L}\right)$ & $1.585(1.060-2.371)$ & $0.025^{*}$ & $0.868(0.522-1.444)$ & 0.586 \\
\hline \multicolumn{5}{|l|}{ Pre-treatment EO } \\
\hline$\left(>0.090 \times 10^{9} / \mathrm{L}\right.$ or $\left.\leq 0.090 \times 10^{9} / \mathrm{L}\right)$ & $0.924(0.618-1.382)$ & 0.702 & - & - \\
\hline \multicolumn{5}{|l|}{ Pre-treatment BA } \\
\hline$\left(>0.010 \times 10^{9} / \mathrm{L}\right.$ or $\left.\leq 0.010 \times 10^{9} / \mathrm{L}\right)$ & $1.151(0.775-1.709)$ & 0.486 & - & - \\
\hline \multicolumn{5}{|l|}{ Pre-treatment total WBC } \\
\hline$\left(>5.361 \times 10^{9} / \mathrm{L}\right.$ or $\left.\leq 5.361 \times 10^{9} / \mathrm{L}\right)$ & $2.023(1.329-3.081)$ & $0.001^{* *}$ & $1.751(1.045-2.935)$ & $0.033^{*}$ \\
\hline \multicolumn{5}{|l|}{ Post/pre-treatment LY ratio } \\
\hline$(>1$ or $\leq 1)$ & $0.839(0.563-1.249)$ & 0.387 & - & - \\
\hline \multicolumn{5}{|l|}{ Post/pre-treatment MO ratio } \\
\hline$(>1$ or $\leq 1)$ & $1.036(0.703-1.527)$ & 0.860 & - & - \\
\hline \multicolumn{5}{|l|}{ Post/pre-treatment NE ratio } \\
\hline$(>1$ or $\leq 1)$ & $0.871(0.571-1.329)$ & 0.521 & - & - \\
\hline \multicolumn{5}{|l|}{ Post/pre-treatment EO ratio } \\
\hline$(>1$ or $\leq 1)$ & $1.180(0.796-1.748)$ & 0.410 & - & - \\
\hline \multicolumn{5}{|l|}{ Post/pre-treatment BA ratio } \\
\hline$(>1$ or $\leq 1)$ & $1.006(0.638-1.585)$ & 0.980 & - & - \\
\hline \multicolumn{5}{|l|}{ Post/pre-treatment total WBC ratio } \\
\hline$(>1$ or $\leq 1)$ & $0.766(0.508-1.154)$ & 0.202 & - & - \\
\hline
\end{tabular}

${ }^{*} P<0.05 ;{ }^{* *} P<0.01$

BA predicted tumor growth. In the present study, chemotherapy down-regulated BA status. While, pre-treatment level and post/pre-therapeutic ratio of BA had no significant effects on OS.Taken together, our present investigation showed that higher pre-treatment level of NE and total WBC counts were correlated with worse prognosis in resectable GC. These noninvasive, simple and low-cost biomarkers may be prognostic indicators. The limitations of our study may include its retrospective design, recruitment of patients from single center, and insufficiency of case number. To eliminate the differences in the general performance of patients, we studied 
each case thoroughly and excluded patients with chronic diseases such as inflammatory bowel diseases and rheumatic diseases, etc.al. Eventually, only a limited number of 104 cases were obtained. Likely, our results were consistent with the previous studies, and we believed our results were highly reliable. Of course, we would conduct a multi-center study and collect more cases data to further explore the conclusion in the future.

\section{Conclusions}

Our study demonstrated that pre-treatment NE and total WBC counts were negatively correlated with overall survival (OS). Surgery significantly decreased the level of NE count, and adjuvant chemotherapy significantly decreased the level of BA. Univariate analysis indicated that AJCC stage (III) and higher level of pre-treatment total WBC count were prognostic factors affecting OS. Multivariate Cox regression analysis revealed that AJCC stage (III) and higher level of pre-treatment total WBC count were independent prognostic factors. Therefore, we suggested that pre-treatment $\mathrm{NE}$ count and pre-treatment total WBC count may be potential prognostic factors for the prognostic evaluation of GCs.

\section{Abbreviations \\ AJCC: The American Joint Committee on Cancer; BA: Basophil; CT: Computed tomography; EDTA: Ethylenediaminetetraacetic acid; EO: Eosinophil; GCs: Gastric cancers; HP: H. pylori; LY: Lymphocyte; MO: Monocyte; NE: Neutrophil; OS: Overall survival; RECIST: Response Evaluation Criteria in Solid Tumors; TAM: Tumor-associated macrophage; TILs: Tumor-infiltrating lymphocytes; TNM: Tumor-nodulus-metastases; WBC: White blood cell}

\section{Funding}

This work was supported by the National Natural Science Foundation of China (grant numbers 81472296, 81602091, 81402176, 81402093, 81272542, 81200369), the Six Major Talent Peak Project of Jiangsu Province (grant number 2015-WSN-022), the Project of Invigorating Health Care through Science, Technology and Education, Jiangsu Provincial Medical Youth Talent (grant number QNRC2016709), the Project of Jiangsu Provincial Commission of Health and Family Planning (grant number H201518), the Science and Education for Health Foundation of Suzhou for Youth (grant number kjxw2015003), the Science and Technology Foundation of Suzhou Xiangcheng (grant nos. SZXC2012-70 and XJ201451) and the Science and Technology Project Foundation of Suzhou (grant numbers SYS201464 and SYS201504).

\section{Availability of data and materials}

The datasets used and/or analysed during the current study are available from the corresponding author on reasonable request.

\section{Authors' contributions}

LW, MT and CK designed the study. WYL, WY and XMD carried out the extraction of data. WMY, WWJ,SLM and GFR carried out the statistical analysis of studies. GXX wrote the paper. All authors read and approved the final manuscript.

\section{Ethics approval and consent to participate}

Ethics approval for the study was granted by the Medical Ethics Committees of the First Affiliated Hospital of Soochow University, and details can be provided by the corresponding author on reasonable request. Consent to participate is not applicable.

\section{Competing interests}

The authors declare that they have no competing interests.

\section{Publisher's Note}

Springer Nature remains neutral with regard to jurisdictional claims in published maps and institutional affiliations.

\section{Author details}

'Department of Oncology, the First Affiliated Hospital of Soochow University, Suzhou 215006, China. ${ }^{2}$ Department of Hematology, the First Affiliated Hospital of Soochow University, Suzhou 215006, China. ${ }^{3}$ PREMED Key Laboratory for Precision Medicine, Soochow University, Suzhou 215021, China. ${ }^{4}$ Department of Radio-Oncology, Nanjing Medical University Affiliated Suzhou Hospital, Suzhou 215001, China. ${ }^{5}$ Department of Oncology, the First Affiliated Hospital of Zhejiang Chinese Medicine University, Hangzhou 310006, China. ${ }^{6}$ Comprehensive Cancer Center, Suzhou Xiangcheng People's Hospital, Suzhou 215000, China.

Received: 25 January 2018 Accepted: 30 May 2018

Published online: 28 June 2018

\section{References}

1. Karimi P, et al. Gastric cancer: descriptive epidemiology, risk factors, screening, and prevention. Cancer Epidemiol Biomark Prev. 2014;23(5):700-13.

2. Siegel RL, Miller KD, Jemal A. Cancer statistics, 2017. CA Cancer J Clin. 2017; 67(1):7-30.

3. Wang C, et al. DBGC: a database of human gastric Cancer. PLoS One. 2015; 10(11):e0142591.

4. Hussain SP, Harris CC. Inflammation and cancer: an ancient link with novel potentials. Int J Cancer. 2007;121(11):2373-80.

5. Su MC, Cheng CY, Wang PC. A neural-network-based approach to white blood cell classification. ScientificWorldJournal. 2014;2014:796371.

6. Wang $M$, et al. Postoperative low absolute lymphocyte counts may predict poor outcomes of hepatocellular carcinoma after liver resection. Chin Med J. 2016;129(5):536-41.

7. Romano F, et al. Immunodeficiency in different histotypes of radically operable gastrointestinal cancers. J Exp Clin Cancer Res. 2004;23(2):195-200.

8. Sanford $\mathrm{DE}$, et al. Inflammatory monocyte mobilization decreases patient survival in pancreatic cancer: a role for targeting the CCL2/CCR2 axis. Clin Cancer Res. 2013;19(13):3404-15.

9. Singel KL, Segal BH. Neutrophils in the tumor microenvironment: trying to heal the wound that cannot heal. Immunol Rev. 2016;273(1):329-43.

10. Li QQ, et al. Neutrophil count and the inflammation-based Glasgow prognostic score predict survival in patients with advanced gastric cancer receiving first-line chemotherapy. Asian Pac J Cancer Prev. 2014;15(2):94550.

11. Schernberg A, et al. Leukocytosis and neutrophilia predict outcome in locally advanced esophageal cancer treated with definitive chemoradiation. Oncotarget. 2017;8(7):11579-88.

12. Wang SC, et al. Pretreatment neutrophil to lymphocyte ratio independently predicts disease-specific survival in Resectable gastroesophageal junction and gastric adenocarcinoma. Ann Surg. 2016;263(2):292-7.

13. Sakkal S, et al. Eosinophils in Cancer: Favourable or Unfavourable? Curr Med Chem. 2016;23(7):650-66.

14. Karasuyama $\mathrm{H}$, et al. Nonredundant roles of basophils in immunity. Annu Rev Immunol. 2011;29:45-69.

15. Karasuyama H, Yamanishi Y. Basophils have emerged as a key player in immunity. Curr Opin Immunol. 2014;31:1-7.

16. Siracusa MC, et al. Basophils and allergic inflammation. J Allergy Clin Immunol. 2013;132(4):789-801. quiz 788

17. Erlinger TP, Muntner P, Helzlsouer KJ. WBC count and the risk of cancer mortality in a national sample of U.S. adults: results from the second National Health and nutrition examination survey mortality study. Cancer Epidemiol Biomark Prev. 2004;13(6):1052-6.

18. Lee YJ, et al. White blood cell count and the risk of colon cancer. Yonsei Med J. 2006:47(5):646-56.

19. lida $\mathrm{M}$, et al. White blood cell count and risk of gastric cancer incidence in a general Japanese population: the Hisayama study. Am J Epidemiol. 2012; 175(6):504-10

20. Yamaoka Y, et al. Induction of various cytokines and development of severe mucosal inflammation by cagA gene positive helicobacter pylori strains. Gut. 1997;41(4):442-51.

21. Wozniak $A$, et al. Interleukin-8 primes human neutrophils for enhanced superoxide anion production. Immunology. 1993;79(4):608-15. 
22. Shen Z, Wu W, Hazen SL. Activated leukocytes oxidatively damage DNA, RNA, and the nucleotide pool through halide-dependent formation of hydroxyl radical. Biochemistry. 2000;39(18):5474-82.

23. Ohshima $\mathrm{H}$, et al. Prevention of human cancer by modulation of chronic inflammatory processes. Mutat Res. 2005;591(1-2):110-22.

24. Karttunen TJ, Niemela S, Kerola T. Blood leukocyte differential in helicobacter pylori infection. Dig Dis Sci. 1996;41(7):1332-6.

25. Ownby HE, et al. Peripheral lymphocyte and eosinophil counts as indicators of prognosis in primary breast cancer. Cancer. 1983;52(1):126-30.

26. Clark EJ, et al. Preoperative lymphocyte count as a prognostic factor in resected pancreatic ductal adenocarcinoma. HPB (Oxford). 2007;9(6):456-60.

27. Muers MF, Shevlin P, Brown J. Prognosis in lung cancer: physicians' opinions compared with outcome and a predictive model. Thorax. 1996;51(9):894-902.

28. Lee $Y$ Y, et al. Clinical significance of changes in peripheral lymphocyte count after surgery in early cervical cancer. Gynecol Oncol. 2012;127(1):107-13.

29. Iwase $R$, et al. Post-operative lymphocyte count may predict the outcome of radical resection for gallbladder carcinoma. Anticancer Res. 2013;33(8):3439-44.

30. Balkwill F, Mantovani A. Inflammation and cancer: back to Virchow? Lancet. 2001;357(9255):539-45

31. Coussens LM, Werb Z. Inflammation and cancer. Nature. 2002;420(6917): 860-7.

32. Mlecnik B, et al. Histopathologic-based prognostic factors of colorectal cancers are associated with the state of the local immune reaction. J Clin Oncol. 2011;29(6):610-8.

33. Gooden MJ, et al. The prognostic influence of tumour-infiltrating lymphocytes in cancer: a systematic review with meta-analysis. Br J Cancer. 2011;105(1):93-103.

34. Zhang J, et al. Preoperative lymphocyte count is a favorable prognostic factor of disease-free survival in non-small-cell lung cancer. Med Oncol. 2013;30(1):352.

35. Elinav $E$, et al. Inflammation-induced cancer: crosstalk between tumours, immune cells and microorganisms. Nat Rev Cancer. 2013;13(11):759-71.

36. Qi Q, et al. Clinical implications of systemic inflammatory response markers as independent prognostic factors for advanced pancreatic cancer. Pancreatology. 2015;15(2):145-50.

37. Shen $\mathrm{SL}$, et al. Elevated preoperative peripheral blood monocyte count predicts poor prognosis for hepatocellular carcinoma after curative resection. BMC Cancer. 2014;14:744

38. Feng F, et al. Low lymphocyte-to-white blood cell ratio and high monocyteto-white blood cell ratio predict poor prognosis in gastric cancer. Oncotarget 2017;8(3):5281-91.

39. Evani SJ, et al. Monocytes mediate metastatic breast tumor cell adhesion to endothelium under flow. FASEB J. 2013;27(8):3017-29.

40. Galdiero MR, et al. Tumor associated macrophages and neutrophils in cancer. Immunobiology. 2013;218(11):1402-10.

41. Watt $D G$, et al. Neutrophil count is the most important prognostic component of the differential white cell count in patients undergoing elective surgery for colorectal cancer. Am J Surg. 2015;210(1):24-30.

42. Wang J, et al. The clinical significance of tumor-infiltrating neutrophils and neutrophil-to-CD8+ lymphocyte ratio in patients with resectable esophageal squamous cell carcinoma. J Transl Med. 2014;12:7.

43. Mantovani A, et al. Cancer-related inflammation. Nature. 2008;454(7203): 436-44

44. Harbaum L, et al. Peritumoral eosinophils predict recurrence in colorectal cancer. Mod Pathol. 2015:28(3):403-13.

45. Samoszuk MK, et al. Occult deposition of eosinophil peroxidase in a subset of human breast carcinomas. Am J Pathol. 1996;148(3):701-6.

46. van Driel WJ, et al. Tumor-associated eosinophilic infiltrate of cervical cancer is indicative for a less effective immune response. Hum Pathol. 1996;27(9): 904-11.

47. PO DEL, et al. Effect of eosinophil cationic protein on human oral squamous carcinoma cell viability. Mol Clin Oncol. 2015;3(2):353-6.

48. Michalaki V, et al. Serum levels of IL-6 and TNF-alpha correlate with clinicopathological features and patient survival in patients with prostate cancer. Br J Cancer. 2004;90(12):2312-6.

49. Helbig G, et al. Chronic eosinophilic leukemia-not otherwise specified has a poor prognosis with unresponsiveness to conventional treatment and high risk of acute transformation. Am J Hematol. 2012;87(6):643-5.

50. Utsunomiya A, et al. Clinical significance of a blood eosinophilia in adult Tcell leukemia/lymphoma: a blood eosinophilia is a significant unfavorable prognostic factor. Leuk Res. 2007;31(7):915-20.
51. Molin D. Bystander cells and prognosis in Hodgkin lymphoma. Review based on a doctoral thesis. Ups J Med Sci. 2004;109(3):179-228.

52. Davis BP, Rothenberg ME. Eosinophils and cancer. Cancer Immunol Res. 2014;2(1):1-8

53. De Monte $L$, et al. Basophil recruitment into tumor-draining lymph nodes correlates with Th2 inflammation and reduced survival in pancreatic Cancer patients. Cancer Res. 2016;76(7):1792-803.

\section{Ready to submit your research? Choose BMC and benefit from:}

- fast, convenient online submission

- thorough peer review by experienced researchers in your field

- rapid publication on acceptance

- support for research data, including large and complex data types

- gold Open Access which fosters wider collaboration and increased citations

- maximum visibility for your research: over $100 \mathrm{M}$ website views per year

At BMC, research is always in progress.

Learn more biomedcentral.com/submissions 